\title{
Modelling seasonal influences on reactive indoor air pollution chemistry for residential environs in the Southern Hemisphere
}

\author{
S. J. Maisey ${ }^{1}$, S.M. Saunders ${ }^{1}$, N. West ${ }^{2}$, P.J. Franklin ${ }^{1}$ \\ ${ }^{1}$ University of Western Australia, Crawley, Western Australia \\ ${ }^{2}$ ChemCentre, resources \& Chemistry Precinct, Bentley WA \\ Email: maises03@student.uwa.edu.au
}

In the developed world people spend over $80 \%$ of their time indoors. The quality of the air indoors is therefore very important for population health. Indoor air quality may be affected by the photochemical degradation of primary indoor air pollutants. These indoor reactions need to be understood because they lead to secondary pollutant formation, which may have potentially serious and often unknown health implications. A highly detailed computational model study for UK homes proposed that free radical driven chemical degradation of constituents of indoor air contribute significantly to the formation of secondary air pollutants. Furthermore that the reaction pathways for indoor radical formation are different to those of outdoors, being highly dependent on the levels of ozone $\left(\mathrm{O}_{3}\right)$ and reactive monoterpenes (MTs) present (Carslaw 2007).

The study presented here was undertaken to investigate indoor air chemistry of residential properties in Perth, (WA) across two seasons. Observational measurement data from these houses were then used to constrain a near explicit chemical model for indoor environs in the southern hemisphere (SH). This, like Carslaw (2007) is based upon the Master Chemical Mechanism (MCM) (http://mcm.leeds.ac.uk/MCM), as the model framework. Data were collected from 88 homes over two seasons.

The southern hemisphere indoor-air box model was developed based on an extended MCM framework. This was developed to be a more refined and reflective model and to extend what has previously been reported on in the literature. Indoor volatile organic compound (VOC) emission rates were modified to reflect the typical air exchange rate expected for the type of houses sampled. Representative outdoor $\mathrm{O}_{3}$ and NOx profiles were generated using air quality monitoring station (AQMS) data over the sampling period. Output data from this model were evaluated to investigate the seasonal changes in the indoor air chemistry. Here we observed subtle changes in the indoor air chemistry, as a consequence of house functioning (heating and air exchange) resulting from the changing outdoor climate conditions. As this is a baseline model, ongoing sensitivity testing over the range of observations made will give a greater understanding of the influence of reactive indoor air chemistry in comparison to models that consider physical processes alone. This is the first detailed indoor air study in Perth, WA, combining measurements of $\mathrm{O}_{3}, \mathrm{NO}_{2}, \mathrm{VOC}$ and meteorological parameters in this geographic location to then constrain a complex and highly explicit indoor air model.

Keywords: Chemical modelling, reactive chemistry, VOCs, Indoor air pollution 


\section{INTRODUCTION}

Indoor air quality (IAQ) directly influences population health, particularly in developing countries poor IAQ from indoor biomass burning is a major contributor to morbidity and mortality. However, IAQ is also important in the developed world where people spend a very high proportion of their lives indoors. Since the 1970s significant research has been undertaken to understand the factors that contribute to the introduction of air pollutants indoors (Weschler 2009). Secondary pollutants, whose presence in the air is the result of chemical reactions of volatile organic compounds (VOCs), are of high concern for their potential health implications (Sunil et al. 2007). Therefore the photochemical degradation reactions of VOCs indoors need to be studied in detail. Beyond traditional analytical testing, computational modeling is a valuable tool to determine which secondary species may be produced indoors. Carslaw (2007) presented a highly detailed computational model study for UK homes, but it lacked specific observational measurement data. This work proposed that free radical driven chemical degradation of constituents of indoor air contribute significantly to the formation of secondary pollutants. The conclusion being that the driving forces of chemistry indoors are commonly observed compounds. For example, the attenuation of light indoors shifts the primary route for $\mathrm{OH}$ production to favour reactions between $\mathrm{O}_{3}$ and monterpenes/alkenes. Similarly $\mathrm{NO}$ and $\mathrm{O}_{3}$ indoors determines peroxy radical production.

This study intends to create a near explicit (143 VOCs, nearly 17,000 reactions) model adaptable for the specific indoor residential environment being modeled through the inclusion of observational data. Carslaw's (2007) paper highlighted the importance of reducing assumptions for the variables and factors that influence indoor chemistry. The model was developed with the Master Chemical Mechanism (MCM) framework, (http://mcm.leeds.ac.uk/MCM) for the climate, architecture and typical conditions of Western Europe. We have worked to extend this model by reducing the number of assumed variables; through the collection of observed data. Room size, air exchange, population density, climate and VOC sources are highly variable and markedly different in the northern and southern hemispheres and so location specific data is important for model construction. We undertook an investigation of the indoor air chemistry of residential properties in Perth, Western Australia (WA) across two seasons. Observational measurement data from these houses were then used to constrain a near explicit chemical model for indoor environs in the southern hemisphere (SH).

This paper briefly describes the analytical methods used to collect data from a number of Perth metropolitan homes and the subsequent inclusion of this data into a model construct. The output from these models was used to examine the influences that seasonal variations have on the reactive chemistry in the indoor residential environment.

\section{FIELD SAMPLING}

\subsection{Sampling Methodology}

A method for detecting a large number of trace chemical compounds was developed through a series of method validation tests. A customized compound list of certified reference materials (Spex Certiprep, Metuchen NJ) allowed for 52 VOC to be assessed. These were chosen based on the substantial literature reporting of common primary indoor species. Multisorbent thermal desorportion (TD) tubes were chosen because they are highly economical, transportable and reliable with measures taken to ensure that recovery and compound validation fell well within the US-EPA TO-17 compendium method guidelines (USEPA 1999). The Perkin Elmer AirToxics (0.3g) TD tubes were connected to SKC calibrated Pocket Pumps at a measured flow as near to $200 \mathrm{ml} / \mathrm{m}$ as achievable and a $20 \mathrm{~L}$ sample volume to be collected. Sample analysis was conducted on a Perkin Elmer 650 Automatic Thermal Desorber paired with a Varian 4500 GCMS; the limit of detection was typically $0.04-0.06 \mu \mathrm{g} / \mathrm{m}^{3}$.

The data for the observational study were collected from residential homes in Perth, Western Australia (Population; 1.45 million, ABS 2008). Volunteer households were recruited through a mixture of previous health study participants, letter drops and referrals from other volunteers. The location of the majority of homes, in the southern suburbs represented typical Perth suburban living (ABS 2008) see figure 1. In order to assess a broad range of indoor air quality scenarios (low to high concentrations) specific household types were targeted:

- 'Typical' Australian single storey brick residences built within the past 40 years,

- Homes located within $100 \mathrm{~m}$ of a high traffic $\operatorname{road}(>40$ vehicles/day, MainroadsWA(2010) ) (to determine the impact of traffic related pollutants), 
- Homes built or renovated within the past 12 months (which are likely to have elevated levels of indoor VOCs (Brown 2002)).

Some apartment residences were also tested. Indoor and outdoor air measurements were taken as residents carried out their normal routine.

Sampling took place weekdays between 9am and 3pm. Individual sampling periods were 100 minutes within this time frame, with up to 3 houses sampled each day. This was determined to be a non peak traffic period with minimal household activities taking place. Data previously collected over the course of the day from one location showed minimal variation in this time period.

Samples were taken inside the main living space of the home; this was usually an open plan living, kitchen and dining area towards the back of the home. Equipment was set up in an area clear of activity at a height of 1 $1.5 \mathrm{~m}$. Alongside the TD tube/pump configuration were a weather station (Davis Instruments Vantage Pro 2), portable $\mathrm{WO}_{2}$ resistor electronic $\mathrm{O}_{3}$ and $\mathrm{NO}_{2}$ monitors (Aeroqual 500), and $\mathrm{PM}_{2.5}$ Dustrak monitors. The $\mathrm{O}_{3}$, $\mathrm{NO}_{2}$ and dust monitors were set to a 1 minute sample log rate. Identical equipment was placed directly outside the home for concurrent sampling. Where possible this was

Figure 1: Map showing household locations,

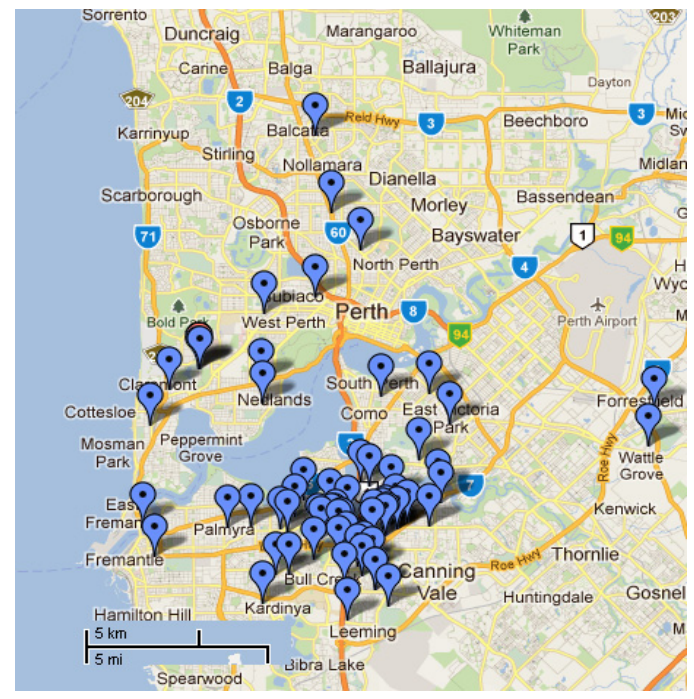
out the front of the home within 5 meters of the front door. However in situations where this was not possible equipment was placed at the back of the home, within 5 metres. A questionnaire was given to all participating households in regards to the house age, location and construction as well as typical chemical, cleaning and ventilation habits and for activities during the sampling period. All participating households were nonsmoking homes.

\subsection{Field Sampling Results}

Table 1. Average indoor VOC concentrations for species observed in $>80 \%$ of samples

\begin{tabular}{|c|c|c|c|c|c|}
\hline Values in $p p b$ & Winter & Summer & & Winter & Summer \\
\hline 2-ethyl toluene & 0.67 & 0.28 & Heptane & 0.63 & 0.43 \\
\hline 4-ethyl toluene & 0.19 & 0.12 & Hexane & 1.1 & 0.47 \\
\hline$\alpha$-terpinol & 0.54 & 0.20 & Limonene & 4.7 & 3.6 \\
\hline Benzene & 1.10 & 0.42 & Nonane & 0.29 & 0.27 \\
\hline Benzene,1,2,4trimethyl & 1.10 & 0.43 & Octane & 0.32 & 0.13 \\
\hline Benzene, ethyl- & 2.10 & 0.31 & Phallandrene & 0.97 & 0.090 \\
\hline cis /trans 2-butene & 13 & 17 & $\alpha$-pinene & 2.4 & 3.50 \\
\hline Cumene & 0.25 & 0.12 & Propylbenzene & 1.4 & 0.21 \\
\hline Cyclohexane & 0.53 & 0.59 & Styrene & 2.6 & 0.15 \\
\hline Cyclohexane, met yl & 0.11 & 0.16 & Toluene & 8.6 & 4.3 \\
\hline Decane & 0.26 & 0.55 & Xylene, m- \& p & 2.8 & 0.62 \\
\hline Ethanol, 2-butoxy- & 0.22 & 0.40 & Xylene, o- & 2.4 & 0.40 \\
\hline Ethene, tetrachloro- & 0.42 & 0.23 & & & \\
\hline
\end{tabular}

Two field campaigns were undertaken; initially in the winter months of June-September 2010 and again in the summer months January-April 2011. Data were collected from 38 homes over the winter period of which 30 were separate homes, and 8 were units or apartments. This is representative of the typical breakdown of Perth housing types (ABS 2008). Ambient temperatures ranged from $11-26^{\circ} \mathrm{C}$ during sampling, with rain observed on 5 of the 21 days samples were taken. Fifty homes (40 separate, 10 units) were sampled in the summer period but VOC data were available only for 31 homes. Ambient temperatures ranged from $29-42^{\circ} \mathrm{C}$ in the summer and there were no days with rain. VOC data were collected from 18 of the homes in both seasons.

Average indoor VOC concentrations were lower in summer compared to the winter testing season (see Table 1) with similar species observed in both seasons. Of the 52 calibrated VOC species 48 were observed in at least $20 \%$ of samples. Benzene, toluene, ethylbenzene, xylene (BTEX), monoterpenes (i.e. pinene, limonene and carene) as well as several alkanes and alkenes were observed in over $80 \%$ of houses (indoors): these are important to note for indoor chemistry. Indoor concentrations were generally 5 or more times those observed outdoors in winter and 3 times greater in summer. These results were comparable to previous Perth VOC studies (Maisey et al. 2009). For $\mathrm{NO}_{2}$ and $\mathrm{O}_{3}$, there were differences in magnitude between homes but both 
consistently peaked towards the early afternoon. Summer outdoor $\mathrm{O}_{3}$ was observed as high as 120 ppb at peak times on a very warm, sunny afternoon. More frequently, peaks of $30-40 \mathrm{ppb}$ were observed. Winter outdoor $\mathrm{O}_{3}$ was significantly lower with a season high of $40 \mathrm{ppb}$ recorded and more usual peaks of $20-25$ ppb. Concentrations of $\mathrm{O}_{3}$ indoors had little seasonal variation, with low levels $(0-6 \mathrm{ppb})$ typically being recorded in both periods. For indoor $\mathrm{NO}_{2}$ typical values peaked at $25-30 \mathrm{ppb}$ in summer with a slightly higher maximum of $30-40 \mathrm{ppb}$ in winter.

\section{INDOOR MODEL STRUCTURE}

A preliminary southern hemisphere indoor-air box model was developed based on the most recent MCM framework. The chemical mechanistic information was taken from the Master Chemical Mechanism, MCM v3.2 (Jenkin et al. 1997, Saunders et al. 2003 for non aromatic schemes; and Jenkin et al., 2003; Bloss et al., 2005 for aromatic schemes), via website: http://mcm.leeds.ac.uk/MCM. The indoor-air box model followed the construct of Carslaw (2007), to reflect a baseline model for the typical Perth home. The MCM v3.2 (143 VOC, 17,000 reactions) now includes the degradation of limonene, which evidence (Carslaw 2007, Forester and Wells 2011) suggests is an important driving agent for indoor air chemistry and had previously only been approximated.

$$
\frac{d C_{i}}{d t}=V_{d}\left(\frac{A_{i}}{V_{i}}\right) C_{i}+\lambda_{r} f C_{o}-\lambda_{r} C_{i} \frac{Q_{i}}{V_{i}}+\sum_{j=1}^{n} R i j
$$

The base model equation (1) was taken from Carslaw (2007), Vd; deposition velocity values were grouped as described in Sarwar et al. (2002). Total house surface area $\left(A_{i}\right)$ and Volume $\left(V_{i}\right)$ was calculated from observations. The Ai and Vi of a typical $3 \times 2$ single storey Perth home with a large open plan living space was $670 \mathrm{~m}^{2}$ and $335 \mathrm{~m}^{3}$, respectively. Indoor $\left(C_{i}\right)$ and outdoor $\left(C_{0}\right)$ VOC concentrations were initially calculated from mean observed values. VOCs for which observation data was not available was nominally set at $0.2 \mathrm{ppb}$. The air exchange values of the home $\left(\lambda_{\mathrm{r}}\right)$ were set to $0.41 \mathrm{~h}^{-1}$ in winter (Haste et al. 2009) and $1.3 \mathrm{~h}^{-1}$ in summer (MFESA 2011). Emissions species $\left(\mathrm{Q}_{\mathrm{i}}\right)$ were calculated as described in Sarwar et al. (2002) using mean indoor/outdoor concentration values for observed species that are included in the MCM. For MCM species not observed in the study initial values were calculated based on values for observed species (with like species being grouped). Reaction rates (Rij) were taken from literature (as described in http://mcm.leeds.ac.uk/MCM/); Complex rates were updated to IUPAC 2009 and Atkinson et al. (2006). Photolysis indoors was treated following Nazaroff and Cass (1986), with the base case initially run with attenuation factors (0.1) of outdoors, with photolysis rates updated using the TUV (Madronich 1998) model tailored for the Perth region seasonal solar declination and zenith angle. Outdoor radical profiles were approximated from available literature for; $\mathrm{OH}, \mathrm{CH}_{3} \mathrm{O}_{2}$ and $\mathrm{HO}_{2}$ and were broadly consistent with field observations (Platt et al. 2002, Emmerson et al. 2005). HONO profile was set $\sim 300$ ppt at night and $\sim 20 \mathrm{ppt}$ during day (Alicke et al. 2003).

\subsection{Indoor Model Output}

Figure 2 Example of 'typical' Temporal change in $\mathrm{O}_{3}$ and $\mathrm{NO}_{2}$ indoors

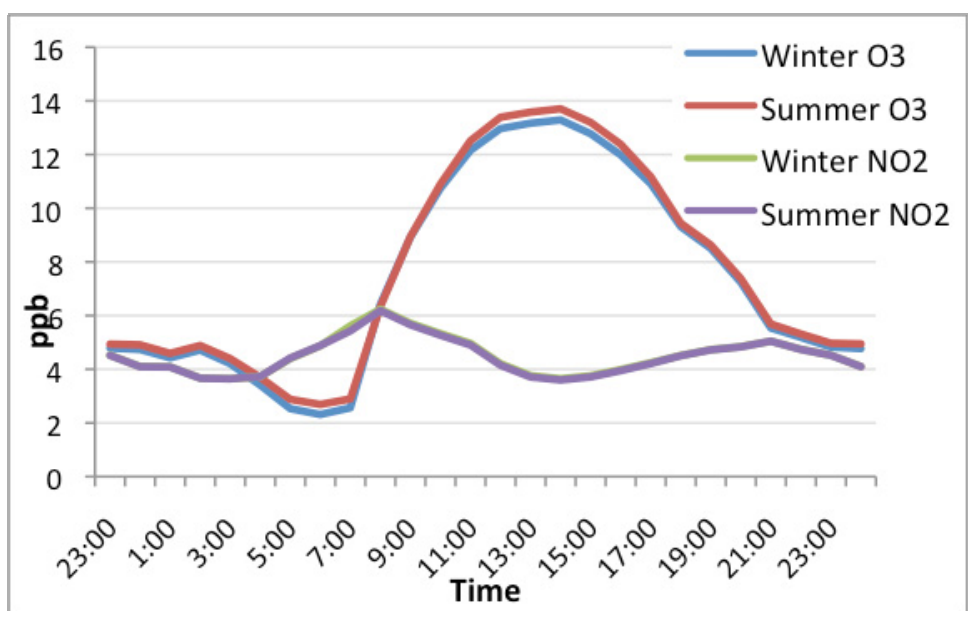

The baseline models were run using the Facsimile 4D software program (mcpa 2011). Each simulation was run for 96 hours with the model output set hourly from 06:00 $\mathrm{h}$. The final 24 hours of output was used for analysis, as this represents a point where near steady state of $\mathrm{OH}$ radical formation is achieved. An example of model output displaying inorganic compound temporal change is shown in Figure 2. Examples of individual VOCs as well as the total hourly change in total VOCs (TVOC) is also presented (figures 3 and 4). 
Figure 3 Individual VOC concentration profiles (ppb)
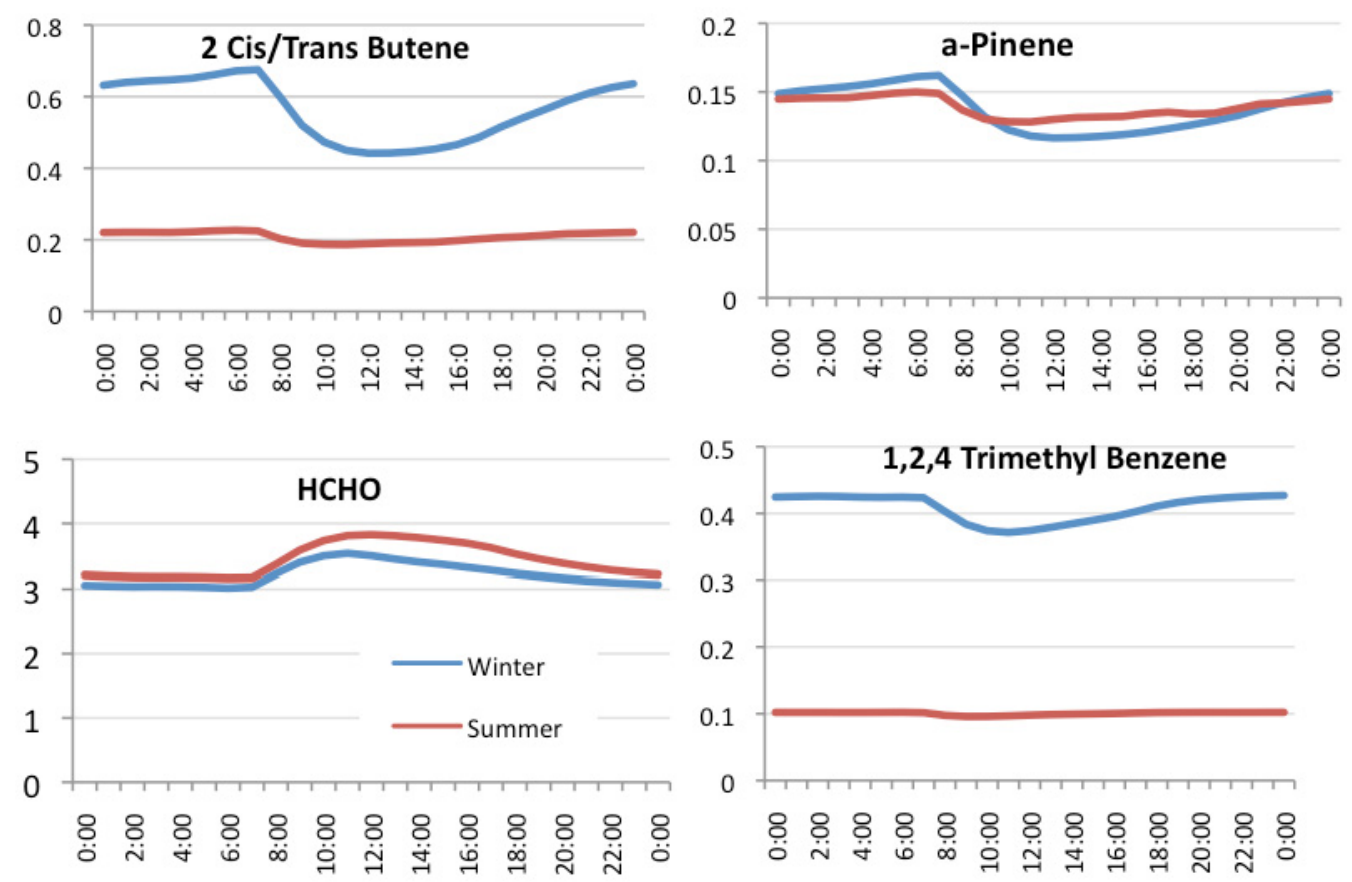

Figure 4 Hourly change in total VOC (TVOC)

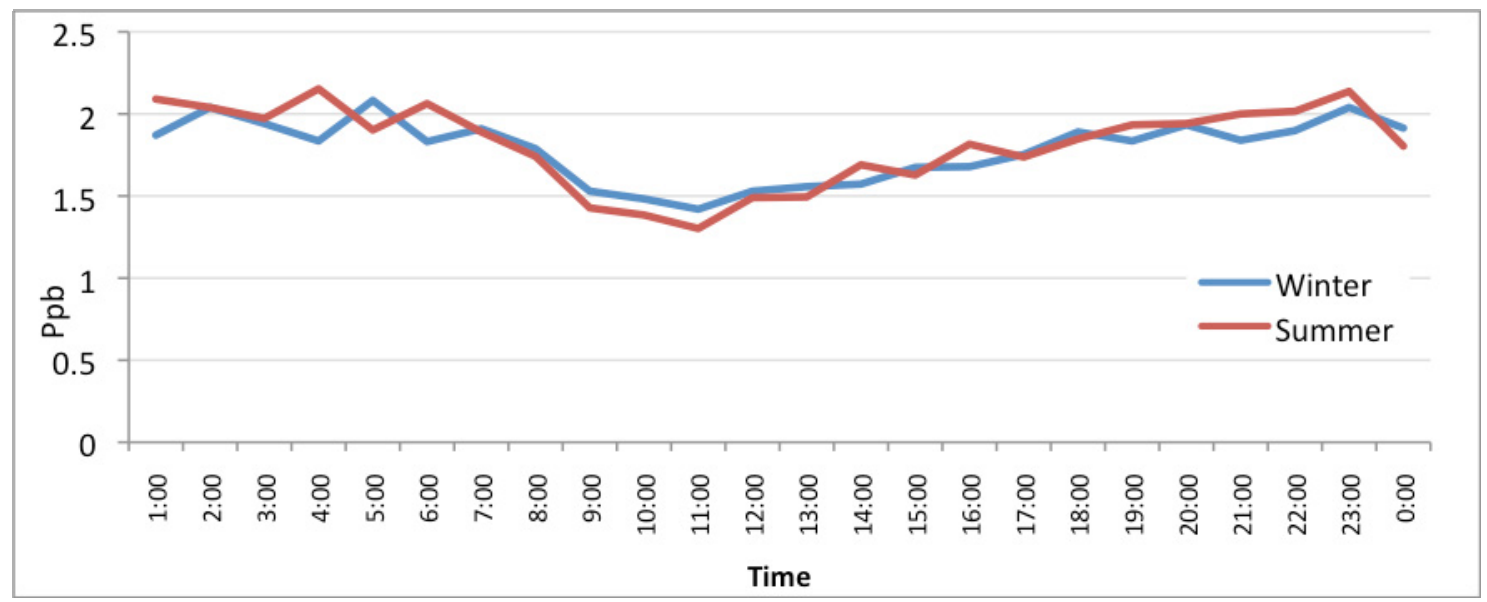

\section{DISCUSSION AND CONCLUSIONS}

Presented in this paper is the preliminary construction model for indoor air reactive chemistry in Perth, WA. The reported data extends Carslaw's (2007) work by including observation specific data and an updated MCM protocol. There are a number of points of interest raised from the results of this preliminary study.

Constraining the model with a more realistic representation of the West Australian indoor VOC composition has begun to give insight into the influence it has on radical and secondary product formation. The order of magnitude and distribution of the $\mathrm{O}_{3}$ and $\mathrm{NO}_{2}$ output from the model (Figure 2) is in agreement with the observed data from homes in this study and this output suggests no obvious seasonal effect indoors. The $\mathrm{OH}$ radical concentrations from the model are also in reasonable agreement with those presented in Carslaw (2007) and follow the expected diurnal variation. The indoor profiles from this base case model are comparable with the observational data; however sensitivity testing on this factor has not yet been carried out. Here it is important to note the high variability of the $\mathrm{O}_{3}$ and $\mathrm{NO}_{2}$ measurement data between houses (see section 2.2), as well as the ambient data from the Western Australian Department of Environment and Conservation (DEC 2011) air monitoring sites. These emphasize the importance of location specific data and 
it will be valuable to perform model sensitivity tests to determine how such variations affect the chemistry of indoor air.

Construction of the summer and winter models included observed data for temperature and humidity. Despite the substantial ambient temperature and humidity differences across the seasons, only a mild $\left(2-5^{\circ} \mathrm{C}\right)$ increase in indoor temperature during summer was observed. The most significant difference between seasons is the larger air exchange rate in the summer months, due to seasonal winds and the greater chance of householders opening windows and doors. With these points in consideration we may note that despite the higher observed indoor VOCs in winter, the hourly change in TVOCs from the model output shows little difference between seasons. Figure 4 shows the trend for change in TVOC, which represents an expected morning low before increasing in the afternoon. Individual species, as given in some examples in figure 3, did show a seasonal difference in both magnitude and temporal variation.

Ongoing refinements of the model will explore the behavior of reactive components in indoor air, specifically the nitrate radicals and their substrates. These in particular have been highlighted (Norgaard 2011) as of extreme importance for future studies. As new research emerges, with regards to surface reactions and deposition velocities for other species the model can readily be adapted to incorporate these results as important factors for indoor chemistry that is not applicable to ambient (outdoor) chemical models. In addition the current photolysis representation does not include reaction factors for the influence of artificial light indoors and needs to be updated to better characterize the as yet undetermined flux of light in the typical residential indoor environment in Perth, WA.

As has been emphasized the model presented in this paper presents the baseline development of an explicit computational model of reactive indoor air chemistry. Future developments with sensitivity testing of the chemical and physical parameters of household micro climates will enable ongoing study on how our behavior indoors has the potential to influence the quality of the air we breathe.

\section{ACKNOWLEDGMENTS}

The project is partially supported by a Cooperative Research Centre (CRC for Asthma) grant, and thanks are due to the MCM development group in Leeds University, UK and J. Loveday who assisted with all the field sampling.

\section{REFERENCES}

ABS, Australian Bureau of Statistics (2008), 2006 Census of Population and Housing Quick Stats: Perth (Major Statistical Region) www.censusdata.abs.gov.au

Alicke B., Geyer, A. Hofzumahaus A., Holland F., Konrad S., Patz H. W., Schafer J., Stutz J., Volz-Thomas A., Platt U. (2003) $\mathrm{OH}$ formation by HONO photolysis during the BERLIOZ experiment. Journal of Geophysical Research 108, 8247

Atkinson R., Baulch D.L., Cox R.A, Crowley J.N, Hampson R.F., Hynes R.G., Jenkin M.E., Rossi M.J., Troe J. (2006) Evaluated kinetic and photochemical data for atmospheric chemistry: Volume II - reactions of organic species. Atmospheric Chemistry and Physics. Discussion, 5, 6295-7168,

Bloss, C., Wagner, V, Jenkin, M.E., Volkamer, R., Bloss, W.J., et al.(2005) Atmospheric Chemistry and Physics, 5, 641-664

Brown S;K. (2002) Volatile Organic Pollutants in New and Established Buildings in Melbourne, Australia Indoor Air ,12, 55-63

Carslaw N. (2007), A new detailed chemical model for indoor air pollution, Atmospheric Environment 41(6) 1164-1179.

DEC (2011), WA Department of Environment and Conservation, air quality monitoring, available at, http://www.dec.wa.gov.au/content/view/3420/2292

Emmerson K.M., Carslaw N., Pilling M.J. (2005) Urban atmospheric chemistry during the PUMA campaign. 2: radical budgets for $\mathrm{OH}, \mathrm{HO}_{2}$ and $\mathrm{RO}_{2}$. Journal of Atmospheric Chemistry 52 (2) 165-183. 
Maisey et al., Modelling seasonal influences of reactive indoor air pollution

Forester C.D, Wells J. (2011), Hydroxyl Radical Yields from Reactions of Ozone with Terpene Mixtures. Proc. $14^{\text {th }}$ International Conference Indoor Air 2011, Texas, USA

Haste H., Moran S., Wilkinson S., James E. (2009). Air exchange rates in Australian homes Proc. $27^{\text {th }}$ Conference Australian Institute of Occupational Hygienists '09 Canberra.

IUPAC (2009) Evaluated kinetic data, available from http://www.iupac-kinetic.ch.cam.ac.uk/

Jenkin, M.E., Saunders, S.M, Pilling, M.J. (1997) The tropospheric degradation of volatile organic compounds: A protocol for mechanism development. Atmospheric Environment, 31, 81-107

Jenkin M.E., Saunders, S.M., Wagner, V., Pilling, M.J. (2003) Protocol for the development of the master chemical mechanism MCMv3 (Part B): Tropospheric degradation of aromatic volatile organic compounds. Atmospheric Chemistry and Physics, 3, 181-193

Madronich, S. (1998). "NCAR/ACD TUV: Tropospheric Ultraviolet \& Visible Radiation Model ", available from http://cprm.acd.ucar.edu/Models/TUV/.

MainroadsWA (2010) Metropolitan Traffic Digest 2003/2004 - 2008/2009, WA government publication available from; www.mainroads.wa.gov.au

Maisey S., Franklin P., West N., Saunders S.M. (2009) Assessment of the indoor/outdoor relationship of VOCs in residential properties in Perth, Western Australia. 19th International Clean Air and Environment Conference by the Clean Air Society of Australia \& New Zealand (CASANZ) ISBN: 9780-9806045-1-1

Mcpa software (2011) http://www.mcpa-software.com/ovframe.html

Metropolitan Fire and Emergency Service Board (MFESA) (2011) A best practice approach to shelter in place for Victoria, p 28. http://www.cfa.vic.gov.au/documents/sip_bestpractice.pdf

Nazaroff, W.W. and Cass, G.R. (1986) Mathematical modelling of chemically reactive pollutants in indoor air. Environmental Science and Technology. 20: 924-934

Nøjgaard, J.K. (2010) Indoor Measurements of the sum of the nitrate radical, $\mathrm{NO}_{3}$, and dinitrogen pentoxide, $\mathrm{N}_{2} \mathrm{O}_{5}$ in Denmark. Chemosphere, 79, 898-904

Platt U., Alicke B., Dubois R., et al. (2002) Free radicals and fast photochemistry during BERLIOZ. Journal of Atmospheric Chemistry, 42, 359-394

Sarwar G., Corsi R., Kimura Y., Allen D., Weschler C.J. (2002) Hydroxyl radicals in indoor environments. Atmospheric Environment, 36 3973-3988.

Saunders S.M., Jenkin, M.E., Derwent, R.G., Pilling, M.J. (2003) Protocol for the development of the master chemical mechanism MCMv3 (Part A): Tropospheric degradation of non-aromatic volatile organic compounds. Atmospheric Chemistry and Physics, 3, 161-180

Sunil V.R., Laumbach R.J., Patel K.J.,, Turpin B.J., Lim H., Kipen H.M., Laskin J.D. Laskin D.L (2007) Pulmonary effects of inhaled limonene ozone reaction products in elderly rats. Toxicology and Applied Pharmacology, 222, 211-220

United States Environmental Protection Agency (USEPA) (1999) Compendium Method TO-17; determination of volatile organic compounds in ambient air using active sampling onto sorbent tubes $2^{\text {nd }}$ Ed. EPA/625/R-96/010b.

Weschler C.J. (2009) Changes in indoor pollutants since the 1950's. Atmospheric Environment, 43, 153-169 